\title{
Análisis de la incidencia del trabajo realizado por los asesores específicos en las Escuelas Unidocentes
}

\author{
Analysis of the incidence of the job done by the specific advisers in unique \\ schools
}

\author{
Luis Alfredo Mendoza Mendoza \\ Ministerio de Educación Pública, Costa Rica \\ luimen123@hotmail.com
}

Recibido: 11 octubre 2010 Aceptado: 12 octubre 2010 Corregido: 28 junio 2011

\section{Resumen}

El estudio aborda la perspectiva de las personas que ocupan el cargo de Asesores de las áreas de Español, Matemáticas, Estudios Sociales y Ciencias y del profesorado unidocente acerca de sus estrategias de gestión y los aportes pedagógicos, para fortalecer el proceso de enseñanza-aprendizaje y en la gestión escolar.

El estudio se llevó a cabo en el circuito escolar 05, Dirección Regional Educativa de Santa Cruz, Costa Rica, en las escuelas unidocentes Palestina, Castilla de Oro, Altos del Roble, Playa Hermosa, Cacique y Nuevo Colón. La investigación fue descriptiva, ya que caracteriza el desempeño de la gestión de los asesores específicos, en beneficio del personal unidocente del circuito en estudio.

Los hallazgos del estudio permitieron identificar que hay carencia de espacios formativos y de desarrollo profesional para el personal que se desempeña en instituciones unidocentes. Por ello, se propone un modelo de gestión, que considera los componentes relacionados con el desempeño de la labor unidocente. El modelo se orienta a la atención de dos grandes áreas de la gestión escolar: el área administrativa y el área pedagógica.

Palabras clave: Educación, Administración de la Educación, Gestión escolar, Supervisión de la educación, Formación docente, Innovación y calidad de la educación.

\begin{abstract}
The study examines the perspective of specific advice from the Spanish areas, math, social studies and science teachers and one teacher of the school circuit 05, Regional Education Directorate Santa Cruz, proper schools: Palestine, Castilla de Oro, Altos del Roble, Playa Hermosa, Cacique and New Colon, on the management carried out by consultants and educational input they expect teachers to strengthen their work in the teaching-learning process, as well as school management.
\end{abstract}


With the present investigation, we identified as a problem or need, lack of training spaces and professional development of teachers one teacher for this reason, we present a management model, which considers the components related to the performance of one teacher's work . The design aims to address two major areas of school management: the administrative and teaching area.

Keywords: education, educational administration, school management, supervision of education, teacher training, innovation and quality of education.

\section{Introducción}

La realidad sociocultural actual exige de las organizaciones educativas la transmisión de conocimientos y la formación de una ciudadanía capaz de reflexión crítica ante los eventos cotidianos. Al respecto, el Estado costarricense, por medio del Ministerio de Educación Pública, lidera la obligación con la sociedad costarricense de ofrecer una educación que satisfaga esas necesidades.

La Ley Fundamental de Educación (1957) en el capítulo I, estipula los fines de la educación costarricense y en el Artículo $2^{\circ}$, indica lo siguiente:

a) la formación de ciudadanos amantes de su Patria, conscientes de sus deberes, de sus derechos y de sus libertades fundamentales, con profundo sentido de responsabilidad y de respeto a la dignidad humana;

b) contribuir al desenvolvimiento pleno de la personalidad humana;

c) formar ciudadanos para una democracia donde se concilien los intereses del individuo con los de la comunidad;

d) estimular el desarrollo de la solidaridad y de la comprensión humanas; y,

e) conservar y ampliar la herencia cultural, impartiendo conocimientos sobre la historia del hombre, las grandes obras de la literatura y los conceptos filosóficos fundamentales. En manos de los educadores se deposita la mayor responsabilidad formativa de las nuevas generaciones. (p.1)

Dentro del esquema educativo, la figura de la persona que administra la educación representa la acción que determina la calidad del proceso educativo; o, por el contrario, la de 
quien se responsabiliza por el fracaso y la mala imagen institucional. Igualmente, la labor docente es fundamental para el logro de la calidad de la educación, pues es en el salón de clases donde se realiza la formación del estudiantado y por consiguiente, la labor más importante del docente.

La persona que administra la institución educativa tiene a su cargo orientar la organización para el accionar y logro de los objetivos educativos. Tanto el éxito administrativo y pedagógico de una institución dependen del desempeño del administrador o administradora educativa. Sin embargo, su labor se ve afectada por de tipo organizativo, curricular, afectivo, económico y otros más; que le obligan a convertirse en una persona profesional con gran variedad de actitudes y destrezas para asumir un liderazgo positivo y permanente.

En Costa Rica, la educación primaria, según la matrícula que ostenten las instituciones educativas, se agrupan en diversas modalidades, entre las que se encuentran las escuelas Unidocentes ${ }^{1}$, cuya matrícula es hasta 30 alumnos. Al respecto, es en las zonas rurales donde las escuelas Unidocentes tienen mayor presencia, ya que se constituyen en el único medio del Estado para cumplir con el mandato constitucional de desarrollar el proceso de enseñanza y aprendizaje y, por tanto, de llevar a cabo la transferencia de la cultura y la formación para la vida en sociedad.

Desde la creación de los centros de enseñanza unidocentes, que empezó en la década de 1940, se presentan una serie de dificultades y limitaciones que los han puesto bajo análisis en diversos estudios, donde se encontraron evidencias de limitaciones importantes en las condiciones mínimas para garantizar la calidad del proceso educativo. De esta manera, se amplían las desigualdades sociales y prevalecen mejores oportunidades para la educación urbana, en detrimento de sectores vulnerables de la población rural.

Alvarado (citada por Torres y Céspedes, 2005), indica que las limitaciones de infraestructura, recursos y mobiliario impiden el desarrollo efectivo de procesos de enseñanza y aprendizaje. Además, los educandos de zonas rurales tienen un 60\% más de probabilidades de repetir el curso lectivo que los del área urbana. Al respecto, Bustos, Campos, Corea, González y Mendoza (2006) señalan que los educadores y educadoras unidocentes deben

${ }^{1}$ En la actualidad, según Resolución DG-0972009, de la Dirección General de Servicio Civil, área de carrera docente, se le denomina a este profesional profesor de enseñanza unidocente. A este profesional le corresponde atender todos los niveles de la educación primaria en forma simultánea y en un mismo salón de clase. 
impartir lecciones en un ambiente multigrado, para el cual no fueron formados en las universidades.

El Manual Descriptivo de Clases de Puestos Docentes del Régimen del Servicio Civil, Decreto Ejecutivo 35513 (MEP, 2005) tipifica al Asesor Pedagógico como un funcionario del Ministerio de Educación Pública, que pertenece al Departamento de Asesoría Pedagógica. Las tareas que realiza este personal son de planificación, asesoramiento, orientación y cualquier otra actividad, vinculada al desempeño técnico de la educación nacional; es decir, se encarga de la ejecución de labores de asesoramiento pedagógico en una determinada especialidad de la enseñanza o en el desarrollo de programas de capacitación y perfeccionamiento del personal docente y administrativo docente.

Debido a la particularidad de las escuelas unidocentes, que se hace referencia en esta investigación, se estudiaron las funciones y el rol de los Asesores Específicos ${ }^{2}$ de las áreas básicas de Español, Ciencias, Estudios Sociales y Matemática, y el grado de incidencia de su trabajo en el desempeño de las funciones docente-administrativas de maestros y maestras unidocentes del circuito 05, de la Dirección Regional Educativa de Santa Cruz, con el propósito de conocer si los Asesores Específicos, en cuanto a la dotación de herramientas metodológicas y pedagógicas, facilitan su labor diaria en el aula.

\section{Antecedentes}

El rol de los Asesores Específicos y su aporte a la formación de los maestros y maestras unidocentes es un tema poco investigado; por tal razón, no existe suficiente información al respecto.

En investigaciones realizadas por autores nacionales e internacionales, se aborda la temática de las funciones de los Asesores Supervisores, relacionadas, principalmente, con la gestión escolar, la práctica pedagógica, la administración y la gestión educativa, el desarrollo del currículo, la calidad educativa y la supervisión educativa, pero no en relación con sus aportes al personal unidocente.

\footnotetext{
${ }^{2}$ Mediante Decreto 35513-MEP, este funcionario se denomina, en la actualidad, Asesor Regional.
} 
En el nivel nacional, se encontraron estudios de escuelas unidocentes ligados principalmente, a las siguientes temáticas: gestión escolar, cogestión educativa y organizacional, gestión del asesor supervisor, entre otros. Con base en la revisión de literatura, se determinó, que la bibliografía disponible hace referencia al papel del Asesor Supervisor, ya que son funcionarios que velan por el funcionamiento técnico administrativo de las instituciones educativas; es decir, que tienen a su cargo aspectos de carácter administrativos, por lo que esta población no se incluyó en el estudio.

\section{Justificación de la investigación}

La responsabilidad de administrar una institución educativa, por pequeña que sea, requiere de habilidades y destrezas personales y profesionales, así como de motivación y compromiso de parte del director o directora. Como el conocimiento teórico y técnico, la actitud que el administrador de la educación interponga en sus acciones administrativas es determinante en la calidad de la gestión institucional.

Esta investigación se enfocó en dos aspectos de la realidad educativa costarricense de gran relevancia: el desempeño del personal docente unidocente, que es una población mayoritaria del sector educativo a nivel nacional. Al respecto, en el 2008, había 1800 escuelas unidocentes y, por lo general, están ubicadas en zonas rurales (CONARE, 2011).

El otro aspecto analiza la labor que realizan los Asesores Específicos de las Áreas Básicas (Español, Matemáticas, Estudios Sociales y Ciencias) de la Dirección Regional Educativa de Santa Cruz, ya que entre sus funciones está asesorar al personal docente, mediante diferentes propuestas metodológicas y materiales acordes con los contenidos de los programas de estudio oficiales. Por consiguiente, los Asesores Específicos deben promover acciones para atender las necesidades de educandos y docentes; $y$, en este caso, al maestro o la maestra unidocentes, quienes enfrentan las mayores limitaciones para el ejercicio de sus funciones pedagógicas y carencias significativas de recursos humanos, financieros y físicos. 


\section{Problema de investigación}

En el territorio nacional existen veintisiete direcciones regionales educativas y en cada una de ellas hay Asesores Específicos. Si bien no es comparable el accionar de esas direcciones, al existir en un mismo sistema educativo con características homogéneas, se asume que están inmersas en una realidad burocrática (papeleo administrativo, escasez de recursos, entre otros), que es común a todas las ellas. Por tanto, es importante conocer los resultados de la investigación en la Dirección Regional de Santa Cruz, para orientar futuras investigaciones en otras regionales educativas, relacionadas con la formación pedagógica de los del profesorado unidocentes.

En los distintos programas de formación universitaria de formadores no se prepara a la población graduada para laborar en escuelas unidocentes, porque no basta con recibir metodologías sobre cómo abordar el proceso de enseñanza aprendizaje, sino que se requiere conocer la complejidad de las funciones organizacionales y administrativas, el liderazgo y la gestión escolar, entre otras, que deberá asumir el maestro o maestra unidocentes. Ante esta realidad, la función asesora de los Supervisores y Asesores Específicos es de vital importancia, pues tienen el deber de atender las carencias o debilidades de este personal docente en el proceso pedagógico-administrativo propio de los centros educativos unidocentes.

A partir de la realidad descrita de los centros unidocentes, surgió el siguiente problema de investigación:

¿Cómo inciden los Asesores Específicos en el desempeño docente de los maestros y maestras unidocentes?

Con base en el problema de investigación, se formulación los objetivos del estudio:

\section{Objetivos Generales:}


1. Analizar la incidencia de la gestión de los Asesores Específicos, en el desempeño de maestros y maestras unidocentes del circuito 05, Dirección Regional Educativa de Santa Cruz.

2. Diseñar una propuesta de gestión administrativa, acorde con los resultados del estudio que contribuya a mejorar el desempeño de la labor de los personal docente unidocente

\section{Objetivos Específicos:}

1. Identificar las funciones de los Asesores Específicos, según establece el Manual Descriptivo de Clases de Puestos Docentes.

2. Identificar las funciones del personal unidocente, según el Manual Descriptivo de Clases de Puestos Docentes.

3. Comparar las funciones que desempeñan los Asesores Específicos con las tipificadas en el Manual Descriptivo de Clases de Puestos Docentes.

4. Cotejar las funciones que desempeña el personal unidocente con los deberes especificados en el Manual Descriptivo de Clases de Puestos Docentes.

5. Conocer los aportes de los Asesores Específicos en los procesos de gestión del personal unidocente.

6. Identificar factores que limitan la gestión docente-administrativa de los Asesores Específicos.

7. Identificar factores que facilitan la gestión docente-administrativa de los Asesores Específicos.

8. Diseñar un modelo de gestión que permita a los Asesores Específicos contribuir en el desempeño del personal unidocentes.

El estudio fue descriptivo y se llevó a cabo en la Dirección Regional de Santa Cruz, ubicada en el cantón del mismo nombre, donde laboran los Asesores Específicos. Esta Regional Educativa abarca los cantones de Carrillo y Santa Cruz, con sus respectivos distritos. Cuenta con un asesor específico en las áreas de Español, Estudios Sociales, Ciencias, 
Matemática, Inglés, Preescolar, Guanacastequidad y además, con una docente encargada de la asesoría de capacitaciones. Para efectos de este estudio se trabaja únicamente cuatro áreas: Estudios Sociales, Español, Ciencias y Matemática. Se estudiaron las instituciones unidocentes del Circuito Escolar 05: Palestina, Altos del Roble, Castillo de Oro, Nuevo Colón, Playa Hermosa y Cacique.

\section{Referente Teórico}

\subsection{Educación}

La educación constituye el canal por donde fluyen los saberes y las destrezas necesarias para potencializar la producción de conocimiento y prácticas. Delors, Mufti, Amagi, Carneiro, Chung, Geremek, y Nanzhao (1996) indican que la educación a lo largo de la vida es la llave para entrar en el siglo XXI. Ese concepto va más allá de la distinción tradicional entre educación primera y educación permanente y coincide con otra noción formulada a menudo: la de sociedad educativa, en la que todo puede ser ocasión para aprender y desarrollar las capacidades del individuo. Delors et al., (1996) sustentan su posición cuando manifiesta que la educación a lo largo de la vida se basa en cuatro pilares: aprender a conocer, aprender a hacer, aprender a vivir juntos, aprender a ser.

Masís (1990), se refiere al concepto de educación como el proceso de trabajo planificado con base en las características y condiciones del entorno que busca el desarrollo integral del individuo a partir de objetivos preestablecidos, permitiéndole la adaptación y transformación de sus condiciones económicas, políticas y sociales.

\subsection{Administración de la Educación}

La Administración Educativa es definida por Ugalde (1979) como el "proceso que, en su relación, comporta varias acciones, encadenadas, como un conjunto coherente y ejecutadas para obtener del sistema educativo el máximo rendimiento posible" (p. 75). Al respecto, es necesario crear y mantener un ambiente en el que las personas laborando en grupo, alcancen 


\section{Gestión Givecuacocon

con eficiencia metas seleccionadas y para esto se requiere planeación, organización, integración de personal, dirección y control.

Desde esta perspectiva, Masís (1990) indica que la Administración de la Educación es un proceso de trabajo planificado de una organización, que busca con la coordinación de recursos humanos y materiales el logro de objetivos de tipo educativo. Garbanzo y Orozco (2007) consideran que "La administración aplicada al campo de la educación conlleva la responsabilidad de conducir adecuadamente los procesos de gestión educativa en los distintos niveles y modalidades de la educación, articulada a un fuerte enfoque pedagógico, para poder alcanzar el desarrollo de los aprendizajes con altos parámetros de calidad” (p. 97). También señalan que: “La Administración de la Educación desde un enfoque de competitividad, debe estar en correspondencia con las necesidades de transformación social y económicas del país; para lo que se requiere de la articulación de estrategias y políticas educativas en conjunto con las políticas del Estado” (p. 99).

En cuanto al desarrollo organizacional de la Administración de la Educación, según Chiavenato (2007) dentro de las organizaciones educativas, y tomando en cuenta sus niveles de acción, se hace necesario fortalecer niveles de autonomía y descentralización de sus recursos, donde la independencia en la toma de decisiones es fundamental

Salas (citada por García, Rojas y Campos, 2002) comenta que la Administración Educativa como disciplina involucra lo que concierne a un proyecto educativo general y a sus directrices administrativas que lo convierten en realidad, en relación con la filosofía institucional: políticas, visión y misión.

Por tanto, la Administración de la Educación se constituye en una disciplina para estudiar la organización y la estructura de los sistemas educativos. Además, permite orientar las funciones de planificación, dirección, coordinación y control en el funcionamiento de un programa e institución.

\section{Administración de la Educación en Costa Rica}

El sistema educativo costarricense, a través del Ministerio de Educación Pública, delega la Administración de la Educación en 27 Direcciones Regionales de Educación, las 
cuales cuentan con dos departamentos: Departamento de Asesoría Pedagógica y el Departamento de Servicios Administrativos y Financieros. Cada una de las Direcciones Regionales está, a su vez, subdividida en unidades territoriales menores que se denominan circuitos escolares y agrupan las instituciones que los comprenden en un número apropiado para la mejor administración y control, según sean definidos por las autoridades del Nivel Central.

La Supervisión del circuito educativo es la entidad regional encargada de administrar un segmento de las instituciones educativas de las diferentes regiones, mediante el asesoramiento técnico educativo y administrativo, en seguimiento de la legislación educativa y la ejecución de los procesos educativos, por medio de las acciones docentes, además de implementar las acciones de coordinación circuital de asesoría e intercambio de experiencias y conocimiento.

Los circuitos escolares están a cargo de un Asesor Supervisor, que debe asesorar a los directores y directoras de las instituciones educativas en la interpretación de la política educativa, los planes, programas y disposiciones emanadas del nivel superior; velar por el adecuado cumplimiento de la legislación atinente y de los deberes y obligaciones del personal docente, técnico y administrativo de su circuito. Además, debe organizar actividades de asesoría, actualización profesional y estimular el intercambio de experiencias entre el personal de las instituciones a su cargo.

\section{Gestión escolar}

La gestión escolar es un proceso mediante el cual se motiva a participar responsable y conscientemente, a los principales implicados en la labor educativa, para tomar decisiones que incidan positivamente en la calidad de los servicios que el centro ofrece (Lastarria, 2008, p. 14).

El quehacer cotidiano del maestro y la maestra unidocentes obliga el abordaje de dos grandes funciones: por una parte, el desarrollo del currículo nacional, es decir realizar las tareas propias de la formación y la pedagogía; por otra, conlleva el desempeño de las tareas de 
la administración de la institución, que implica el manejo de las políticas y normativas que emana el sistema educativo al respecto.

Ante esas dos funciones, el maestro y la maestra unidocentes se convierten en gestores que deben desarrollar estrategias para alcanzar la calidad educativa en su institución. Al respecto, Castillero (2002) indica que la gestión escolar no es más que el conjunto de actuaciones que se desarrollan en el centro como expresión del gobierno institucional, bajo la conducción y responsabilidad del director, para definir sus objetivos y diseñar la naturaleza y desarrollo de las tareas para alcanzarlos. De igual manera, Alvariño (citado por Ogando, 2004) manifiesta que la gestión es un elemento determinante de la calidad y eficiencia de los centros educativos, en especial, en la medida en que se incrementa la descentralización de los procesos relacionados con la toma de decisiones de los sistemas educacionales.

Vargas (2008), indica que la gestión educativa es vista como un conjunto de procesos teórico-prácticos articulados dentro del sistema educativo, en respuesta a los lineamientos institucionales, de manera que la gestión viene a ser una estrategia para comprender y conducir la organización escolar.

\section{Supervisión de la educación}

La supervisión de la educación ha dado origen a innumerables definiciones, Nérici (1975) la define como:

... la expresión máxima del "liderazgo educacional en acción" La Supervisión Escolar apunta al mejoramiento del proceso de enseñanza aprendizaje, para lo cual tiene que tomar encuentra toda la estructura teórica, material y humana de la escuela. (p. 56)

La Supervisión Educativa es el eje que impulsa las acciones de mejoramiento del currículo; su papel fundamental es determinar situaciones, descubrirlas y emitir juicios sobre cómo debe procederse en cada caso; en consecuencia, conlleva el mejoramiento de la instrucción, la evaluación del docente, el liderazgo del currículum y la administración escolar. 
En términos generales, la supervisión es un medio para mejorar. Ocurre cuando se estimula al personal docente a mejorar en forma continua. Esto indicaría claramente que la tarea del supervisor es mejorar la situación de aprendizaje, al realizar una actividad de servicio que apoya a al personal docente para que realice mejor su trabajo.

\section{Formación docente}

La formación inicial de docentes está a cargo de las universidades y no hay capacidad, desde la administración, para influir en los contenidos de la formación (reforma educativa). Existe un divorcio en lo que se enseña en las universidades, con lo que se vive en el trabajo cotidiano en los salones de clase. La teoría es una y la práctica es otra. A pesar de que el Ministerio de Educación Pública es el gran empleador, la formación inicial deja lagunas sin cubrir en las expectativas y requerimientos del mismo para los puestos docentes, principalmente en relación con las labores administrativas y de gestión educativa, lo que implica que la formación docente es un proceso que perdura en el tiempo, con la adquisición y actualización de los conocimientos, habilidades y valores oportunos para el desempeño de la labor docente.

Es necesario buscar diversas alternativas para la actualización profesional, que ofrezcan herramientas sólidas para responder a las exigencias del cambio, que permitan responder con eficiencia los retos de la administración de la educación (Vargas, 2008).

\section{Innovación y calidad de la educación}

Aceptar que el cambio es en esencia aprendizaje, tiene una implicación importante. Significa que los centros educativos son lugares de aprendizaje para el profesorado, a partir de su experiencia cotidiana, así como de las actividades y tareas que ejecutan. Se podría inferir que el personal docente que se considera aprendiz en sus clases, será más competente al facilitar el aprendizaje del alumnado. La sensibilidad que se desarrolla en los intentos de aprender nuevas ideas y modos de trabajar repercute en la forma como se enfrenta al alumnado. Al respecto, Palladino (2005), considera que al hablar de calidad, se hace 
referencia a una persona con capacidad de razonamiento abstracto, de juicio crítico para desempeñarse adecuadamente en el campo social, con habilidades para trabajar en equipos y con capacidad para consensuar e interpretar relaciones.

En relación con lo anterior, el Ministerio de Educación Pública (2008) ha trabajado la calidad de la educación en unión con los valores de cada persona, a la cual la sociedad busca satisfacer sus necesidades, lo que concuerda con la etimología de la palabra "bueno” y si algo es bueno, existe seguridad, placer, satisfacción, que es la energía que mueve al ser humano y llevan al placer de servir.

De acuerdo con las características citadas, los logros en materia de calidad educativa que persiga la Administración de la Educación sustentan los objetivos que de la organización. Particularmente, la definición de calidad educativa que adquiera cada entidad del sistema educativo, dependerá de sus necesidades institucionales y comunales.

\section{Metodología}

Por las características de este estudio, la investigación fue descriptiva. Esta investigación aborda la óptica de los Asesores Específicos de las Áreas Básicas (Español, Matemáticas, Estudios Sociales y Ciencias) y la de los maestros y maestras unidocentes del circuito escolar 05, ambos pertenecientes a la Dirección Regional Educativa de Santa Cruz, y se estudia la gestión de los asesores y el aporte pedagógico que esperan de ellos los docentes, para fortalecer su gestión y labores en el proceso de enseñanza y aprendizaje. Es descriptiva, ya que busca puntualizar el desempeño de la gestión de los Asesores Específicos, en beneficio de los maestros y maestras unidocentes en el circuito escolar en estudio.

El estudio descriptivo permite comprender el papel que los asesores deben de cumplir en el desempeño de la gestión de asesoría y formación de los docentes y las expectativas del personal docente unidocentes respecto de esa labor; todo ello, a partir de un análisis de los principios de la Administración de la Educación.

Fue importante conocer la opinión del personal docente unidocente, respecto de la gestión de los Asesores Específicos, como apoyo a la administración de las escuelas 
unidocentes, para ofrecer recomendaciones en pro del mejoramiento del proceso de la gestión educativa y, por ende, de una mayor calidad de las organizaciones educativas.

Dada la cantidad de sujetos que conformaron el universo del estudio no fue necesario trabajar con una muestra, sino con toda la población de los Asesores Específicos y docentes unidocentes del circuito 05. Para recolectar la información se utilizó un cuestionario, que se estructuró en cuatro partes fundamentales: la identificación, la introducción, las instrucciones y el cuerpo o grupo de preguntas. Se utilizaron dos cuestionarios: uno para los Asesores Específicos y otro para el personal docente unidocente.

\section{Sistematización y análisis de la información}

\subsection{Caracterización de la población}

En relación con la formación profesional del personal censado, se encontró que todos son graduados de nivel de bachillerato y licenciatura. Tres de ellos iniciaron sus estudios en un centro universitario público y, luego, ampliaron sus estudios en universidades privadas. Además, dos de ellos manifestaron poseer otros estudios afines a su desarrollo profesional.

En cuanto a la experiencia docente, en su mayoría cuentan con más de quince años de laborar para el Ministerio de Educación Pública y en la institución diez años o más, a excepción de dos docentes. En general, contaban con experiencia unidocente cuando asumieron el puesto en la institución en estudio.

Con respecto al nivel académico de los Asesores Específicos, tres poseen grado de licenciatura y solo una persona indicó tener grado de maestría. La mayoría de los participantes indicó contar con otros estudios afines a la educación; por ejemplo, uso de la tecnología y evaluación.

En cuanto a la experiencia laboral, tres asesores contaban con cuatro o más años de laborar en el Departamento de Asesoría Pedagógica de la Regional Educativa de Santa Cruz. Solo una docente fue nombrada ese año en una de las asesorías.

\subsection{Funciones de los Asesores Específicos}


Para analizar la incidencia de la gestión de los Asesores Específicos en el desempeño del personal docente unidocente del circuito 05 en estudio, se utilizó lo estipulado para ese cargo en el Manual Descriptivo de Clases de Puestos Docentes del Régimen del Servicio Civil (2005). Las personas entrevistadas consideraron que cumplen con todas las funciones que dicta la normativa, relacionadas con el Departamento de Desarrollo Educativo. Sin embargo, indicaron que no realizan las siguientes funciones:

- Desarrollar proyectos de investigación conducentes a la determinación de las necesidades educativas de la región.

- Promover investigaciones sobre aspectos cualitativos o cuantitativos de la educación y su incidencia en la región educativa y proponer las acciones correctivas. (MEP, 2005)

De lo anterior, se desprende que en el área de investigación no se impulsa ninguna acción para identificar las necesidades de la región, ni en aspectos cualitativos o cuantitativos relacionados con el abordaje educativo. El aporte en la gestión educativa con énfasis en las estrategias metodológicas de investigación no existe. Al respecto, en el cumplimiento de las funciones educativas, Mosley, Megginson y Piertri (2005) señalan que: “al infundir coordinación, orden y acción a las tareas, las habilidades administrativas dan apoyo a la habilidad que tienen algunas personas para hacer que las cosas sucedan y que las cosas se hagan” (p. 16).

La Administración de la Educación tiene un papel preponderante en el proceso de enseñanza y aprendizaje, puesto que recae sobre ella el accionar de toda la organización educativa, en lo pedagógico y lo administrativo. Este cometido pierde el horizonte cuando no se brindan los espacios de crecimiento cognitivo y de desarrollo profesional; paradójicamente, las escuelas unidocentes tienden a adolecer más de esta situación de abandono y discriminación. El estudio evidenció que el personal docente unidocente no reciben ninguna capacitación; por el contrario, enfrentan un fuerte control de la gestión escolar. El cumplimiento de la tarea administrativa prevalece en los niveles superiores de jefatura y el 
apoyo en lo pedagógico no representa ninguna prioridad; ante esta situación, cabe preguntarse sobre el concepto de calidad educativa que impulsa el Ministerio de Educación Pública.

Las personas informantes coinciden en que cumplen con las funciones que establece el Manual Descriptivo de Clases de Puestos Docentes del Régimen del Servicio Civil (2005). No obstante, el aporte en investigación no es una de las prioridades de los encargados de ese departamento, ya que manifestaron el recargo de funciones administrativas. En este caso, la planificación y dirección de la gestión educativa, se centra en aspectos de gerencia local y deja de lado el apoyo en materia curricular, pedagógica, administrativa y de recursos en los niveles básicos de la jerarquía educativa. Sobre este tema, Garbanzo y Orozco (2007) coinciden en que los modelos de gestión excesivamente centralistas son un obstáculo dentro de las organizaciones educativas, lo que puede limitar el cumplimiento de distintos proyectos educativos, posición que queda demostrada en la realidad que vive el personal docente unidocente en su desempeño pedagógico y administrativo.

La centralización de las tareas administrativas ha generado un retraso en la dinámica de las organizaciones educativas y las más perjudicadas son las instituciones educativas y mayormente aquellas que no cuentan con el apoyo de la gestión escolar, como es el caso de las escuelas unidocentes.

\subsection{Funciones del personal docente unidocente}

Dentro de la gestión educativa del personal docente unidocente debe realizar una serie de funciones pedagógicas y administrativas, que se indican en el Manual Descriptivo de Clases de Puestos Docentes del Régimen del Servicio Civil (2005). Las personas informantes indicaron que, aunque tienen muchas funciones por cumplir, llevan a cabo las dos tareas encomendadas. No obstante, en la mayoría de los casos, dedican mucho tiempo de trabajo extra, ya que la multiplicidad de funciones no les permite realizarlas en la jornada laboral prevista; entre las actividades extra a su jornada laboral citaron: reunión con los Comités de 
Apoyo, organizar actividades para recaudar fondos, gestionar ante entidades estatales o privadas ayudas para la institución, asistir a reuniones, cumplir con el llenado y entrega de la papelería administrativa, la revisión de los trabajos escolares, la preparación de exámenes y de material de apoyo para estudiantes con necesidades especiales, entre otras. Además, los participantes manifestaron que se les asignan funciones de otras entidades, tales como las del Ministerio de Salud, en diversas campañas de prevención y control de enfermedades.

El Ministerio de Educación Pública (2008), como ente oficial encargado de la planificación educativa del país, obstaculiza el desempeño del personal docente unidocente, cuando recarga de funciones administrativas y no brinda los espacios de asesoría y flexibilidad necesarios para el desempeño administrativo. Esto implica más tiempo y esfuerzo en el cumplimiento de tareas, que van en detrimento del buen desempeño de la gestión escolar. Las organizaciones educativas unidocentes atienden una multiciplidad de funciones que afectan el desarrollo curricular y, por ende, el cumplimiento de los fines de la educación, ya que por distintas razones la carga administrativa impuesta, provoca desatención en el abordaje pedagógico para cumplir con la gama de tareas de índole administrativo y las funciones conexas asociadas a los requerimientos de otras dependencias ministeriales.

El papel del personal docente unidocente debe entenderse como el de la persona encargada de desarrollar el currículo nacional en todos los niveles que la institución ofrece (MEP, 2005). Es decir, la labor pedagógica conlleva atender los seis grados de la Educación General Básica. Al mismo tiempo, se le asigna a este personal la atención administrativa del centro educativo, en igualdad de condiciones de las organizaciones educativas convencionales. Tal situación obliga a realizar la Administración de la Educación en condiciones que, por su complejidad de funciones, requieren de todo el apoyo que se pueda brindar para un mejor desempeño de la gestión escolar, acorde con las políticas nacionales y las necesidades del entorno.

Son muchas las cualidades que las personas profesionales de la educación deben poseer, pero en las escuelas unidocentes, las exigencias aumentan considerablemente. La labor del personal docente unidocente no se centra únicamente en el desarrollo pedagógico; también, la dirección general de la institución y, por tanto, el desarrollo de la gestión escolar son funciones que debe asumir con total. 


\subsection{Funciones que desempeñan en el ejercicio los Asesores Específicos}

La información recopilada permitió identificar que las funciones de los asesores están sujetas al cumplimiento formal; es decir, la práctica de las funciones de asesoría no incide en la gestión de los personal docente unidocente, pues no coincide con sus verdades necesidades. El aporte que hacen estos funcionarios no responde a los requerimientos de la gestión de ese tipo de instituciones.

La realidad educativa evidencia un divorcio entre lo estipulado formalmente y lo que ocurre en la práctica, porque se busca calidad educativa y se da importancia a la educación primaria, pero no se brinda el espacio curricular ni la asesoría adecuada, hay recargo de tareas administrativas y se relega la práctica pedagógica. Al respecto, la Administración de la Educación no cumple su cometido, según lo planteado por Garbanzo y Orozco (2007), quienes mencionan que la administración en los centros educativos lleva implícita la responsabilidad de un liderazgo eficiente en la gestión de la educación, de acuerdo con el enfoque pedagógico.

La función de los Asesores Específicos en el ejercicio profesional se centra en dotar de planeamientos o unidades didácticas a los personal docente unidocente $\mathrm{y}$ en ofrecer capacitación de algún tema en específico, aunque no se indica sobre cuáles temas o ayuda pedagógica se brinda en las capacitaciones. Aquellas necesidades tales como la evaluación de los aprendizajes, metodologías de aprendizaje, técnicas y estrategias de enseñanza, manejo de la disciplina, etc., no se evidencian ser atendidas por estos profesionales tal y como está estipulado en el Manual Descriptivo de Clases de Puestos Docentes del Régimen del Servicio Civil (2005).

\subsection{Funciones que desempeñan en el ejercicio los personal docente unidocente}

Al contrastar las funciones que realiza el personal docente unidocente, con las que indica el Manual Descriptivo de Clases de Puestos Docentes del Régimen del Servicio Civil (2005), las personas entrevistadas señalaron que cumplen con lo estipulado en ese manual y, aún más, ya que al ser los únicos entes educativos de apoyo en las comunidades rurales, recae sobre ellos toda gestión institucional y comunal, además de participar en la búsqueda de 
recursos económicos para solventar las muchas y diversas necesidades de los centros educativos.

Según el último informe del Estado de la Educación (CONARE, 2011), las escuelas unidocentes constituyen el 49,7\% del total de las instituciones públicas de educación primaria, situación que llama la atención porque significa que constituyen casi la mitad de las instituciones educativas del país. No obstante, el apoyo para la gestión educativa de las escuelas requiere de mayor atención, puesto que prestan servicios educativos a un sector de la población con necesidades diversas y complejas, tanto en lo administrativo como en lo pedagógico; por esa razón, dentro del Ministerio de Educación los centros educativos unidocentes deben ser abordados en forma eficiente y brindarles el apoyo necesario.

Acerca de las tareas que mayor atención demandan, la mayoría del personal unidocente consultado indicó que son las tareas administrativas, por la gran complejidad de trámites y papelería que deben entregar diariamente y las constantes salidas que realizan para las diferentes diligencias administrativas que requiere el centro educativo.

La gestión administrativa del personal docente unidocente, se ve limitada debido a las labores pedagógicas. Entre los aspectos que anotaron los entrevistados se citan:

- Atención de varios grados a la vez.

· Falta de tiempo, muchas reuniones y papelería.

- Recargo de la administración educativa.

· Atención y dedicación a las actividades y planeación pedagógica.

Toda organización educativa requiere de un espacio que contemple sus particularidades. Al respecto, Garbanzo y Orozco (2007) afirman que "las organizaciones educativas no se deben dirigir como cualquier empresa, pues las organizaciones educativas difieren del mundo empresarial en lo sustantivo, en razón de ser organizaciones pedagógicas, con un proyecto de sociedad implícito” (p.101).

Cabe resaltar que la brecha social existente entre la educación urbana y la rural es resultado de la deficiente planificación y la mala organización de las reformas curriculares. La ejecución de toda política educativa requiere tomar en consideración las múltiples 
dimensiones del escenario educativo y, por lo tanto, favorecer y garantizar el apoyo necesario a quienes se encuentran en situación de desventaja social.

En el desenvolvimiento pedagógico, la totalidad del personal docente unidocente entrevistado, manifestó que las labores administrativas también interfieren en el desempeño de la labor pedagógica. Así lo expresan:

. “Sí interfiere en mi labor, a veces no se qué hacer. Me estreso demasiado”.

- "Muchísimo, a veces no se qué hacer”.

. "Muchas veces debo asistir a reuniones convocadas por el supervisor y otros”.

- "La atención del centro educativo es igual que cualquier otra institución, sea grande o pequeña. En este caso, el personal docente unidocente debe cumplir conjuntamente labores pedagógicas y administrativas, por lo que el tiempo no alcanza”.

En la función administrativa, el personal docente unidocente encuentra una serie de tareas que demandan mucho tiempo, lo cual conlleva abandonar las labores pedagógicas o a tratar de llevar simultáneamente las dos funciones.

La Junta de Educación y el Patronato Escolar, se constituyen como organismos de apoyo dentro de la gestión educativa de todo centro educativo, pero la mayoría de las personas entrevistadas, manifestó que eso no ocurre debido a las siguientes razones:

- Las personas no se desempeñan en los puestos en los que fueron nombrados y no cumplen con sus deberes.

- Aunque existen estos órganos de apoyo, siempre hay que estar comunicándoles lo que se tiene que hacer.

- No hay iniciativa, y las personas muestran poco interés por las necesidades de la institución, que implica recargo de funciones para el personal unidocente.

Al contar con poca población, en las comunidades donde se encuentran las escuelas unidocentes la situación es difícil, ya que son pocas las personas que se preocupan por trabajar 
para atender las necesidades de la institución. Por consiguiente, el personal unidocente debe asumir las responsabilidades que corresponden a esas instancias; o bien, en la comunidad hay padres y madres de familia que por sus obligaciones y baja escolaridad no pueden llevar a cabo las tareas que el cargo conlleva.

Las personas consultadas manifestaron que prevalece la necesidad de fortalecer la labor pedagógica en los siguientes aspectos:

- Adecuaciones curriculares.

- Evaluación de los aprendizajes

- Metodologías de aprendizaje.

La formación profesional del personal docente unidocente es una de las mayores preocupaciones por lo que el Ministerio de Educación Pública y las universidades deben aunar esfuerzos, a fin de solventar las debilidades de la población docente unidocente en los niveles pedagógico y administrativo.

En su totalidad, el personal docente consultado coincidió en que se brindó apoyo en los planeamientos o unidades didácticas y en menor porcentaje en cuanto a capacitación. Según la información obtenida, no hubo asistencia en evaluación ni en adecuaciones curriculares. Esto evidenciaría que el aporte pedagógico no es el que requiere el personal docente unidocente para una adecuada ejecución del proceso de enseñanza y aprendizaje. Aunque recibieron apoyo en los planeamientos didácticos, se deja de lado el soporte a la innovación y a la investigación.

De igual manera, manifestaron las personas entrevistadas que no recibieron soporte en lo concerniente a la gestión administrativa, de ningún funcionario del Ministerio de Educación Pública. Indicaron que lo han hecho a través de la experiencia y la colaboración de otros docentes y mediante consultas, y que han aprendido por cuenta propia a sacar adelante las tareas de esa índole.

Con respecto a los factores que obstaculizan el trabajo de los Asesores Específicos, se indican tres factores principales, a saber: 
- Falta de tiempo.

- Falta de recursos.

- Pluralidad de funciones.

Las personas consultadas adujeron que se les asignan muchas funciones extracurriculares (informes, papelería, reuniones, etc.), por lo que dejan de lado el apoyo pedagógico. El tiempo destinado a “otras funciones” centra la labor en el trabajo de oficina; así, se pierde de vista el aporte en investigación, la atención de consultas y la producción de insumos pedagógicos. Así mismo, la falta de recursos económicos agrava aún más la eficiencia y eficacia en las labores de toda la organización. En menor grado se señalaron el hacinamiento, las dificultades por razones geográficas y limitantes en la intervención de los Asesores Específicos.

Los sistemas educativos siguen actuando de manera burocrática, por lo que carecen de agilidad para enfrentar los desafíos que se presentan en el contexto en el que se desarrollan, sobretodo con la centralización de funciones en las instancias ministeriales, que limitan el desempeño de la gestión educativa.

Por otra parte, los Asesores Específicos señalan que las tareas administrativas desempeñadas en el Departamento de Asesoría Pedagógica demandan mayor atención, por ejemplo: reuniones de departamento, formulación de proyectos de la región, elaboración de informes, entre otros. Lo contrario sucede con las labores pedagógicas, ya sean asesorías, capacitaciones, visitas a los centros educativos o cualquier otra acción que implique el apoyo a la labor docente. Esto limita el abordaje que los asesores puedan hacer para fortalecer el desempeño docente del personal docente unidocente.

\section{Conclusiones del estudio}

La incidencia de las funciones de los Asesores Específicos en el desempeño pedagógico y administrativo del personal docente unidocente, no es relevante. El aporte en el 
proceso de enseñanza y aprendizaje se limita a proveer de insumos las unidades didácticas, en cuyo caso se entregan a destiempo y con un costo adicional o con el compromiso de ayudar con los materiales didácticos; en este caso, con hojas blancas para fotocopias. Se deja de lado el asesoramiento o capacitación en diversos temas esenciales, no se investiga y, además, se pierde de vista la complejidad de las funciones del personal docente unidocente en las instituciones y comunidades donde se inserta el centro educativo.

La multiciplidad de funciones administrativas que debe el personal docente unidocente, consume el tiempo efectivo que deben dedicar a otras tareas; por ejemplo, la atención individual de sus estudiantes, la preparación de materiales de apoyo en el proceso de enseñanza y aprendizaje, la innovación educativa y los procesos educativos propiamente dichos. También, se desatienden las necesidades educativas individuales. Debido al exceso de trabajo, estos docentes deben llevar a sus hogares parte del trabajo que no se terminó, con lo cual ocupan los espacios de la convivencia familiar para el cumplimiento de la gestión escolar. Dicha práctica se convierte en un vicio profesional, por lo que el espacio íntimo familiar se ve afectado por las funciones que debió concretar en la institución. Así transcurre el diario vivir de del personal docente unidocente.

En cuanto a las funciones de los Asesores Específicos, se concentran en labores de oficina. Se aduce la carencia de recursos y espacio, además un exceso de funciones administrativas, tales como reuniones, llenado de informes y papelería diversa. También, apoyan los programas de la Región Educativa de Santa Cruz y son parte de la dependencia del Departamento de Asesoría Pedagógica, el cual responde al cumplimiento de las políticas de la Regional Educativa.

En síntesis, el ligamen de los Asesores Específicos con el desenvolvimiento formativo del personal docente unidocente es limitado o nulo. La ayuda pedagógica no se brinda como debiera, y no existen oportunidades para la investigación de las necesidades educativas de la región ni de las instituciones educativas. Tampoco se desarrollan programas tendientes al mejoramiento profesional del personal docente unidocente, en detrimento de su labor, y no se toma en cuenta la realidad educativa e institucional este tipo particular de instituciones educativas. 
En este sentido, y con una visión de compromiso y cambio, la Administración de la Educación en todos sus niveles, tiene el compromiso de conducir a los miembros de la organización educativa, a un replanteamiento de los fines y propósitos educativos individuales y colectivos. Hacer del cambio un movimiento que permita atender las necesidades de quienes tienen a su cargo los procesos de enseñanza y aprendizaje, conlleva asumir un carácter independiente en la toma de decisiones y, en consecuencia, mayor autonomía para ese propósito y para asumir las consecuencias de las decisiones tomadas.

Del análisis de la realidad educativa del personal docente unidocente del circuito 05, se evidenció como problema la falta de apoyo pedagógico por parte de los Asesores Específicos; es decir, el trabajo de los asesores no impacta en el desempeño de los personal docente unidocente en una de las áreas fundamentales de su desempeño laboral: la práctica pedagógica.

\section{Desarrollo profesional del personal docente unidocente}

En manos de quienes administran la educación recae gran parte de la responsabilidad de la calidad del proceso educativo. En este sentido, la Administración de la Educación está al servicio de sus propósitos e ideales y no para servirse a sí misma. Por tanto, el liderazgo del administrador de la educación es el que logra que la organización educativa vaya con buen norte y realice los ajustes requeridos hacer en todo el proceso. De esta manera, el cumplimiento de los objetivos propuesto por las organizaciones educativas, permitirán dirigir la educación, según los fines educativos que la sociedad necesita.

En el sistema educativo costarricense existen variados niveles y modalidades de la educación, atendidos por diferentes profesionales que se desempeñan como administradores de la educación, responsables de conducir procesos educativos en aras de la puesta en marcha de los fines y objetivos de la educación costarricense. Entre estos se encuentran: Director(a) Regional, Director(a) del Departamento de Asesoría Pedagógica, Director(a) del Departamento de Servicios Administrativos y Financieros, Supervisores de Centros Educativos, Asesores Pedagógicos, Directores de las instituciones y los personal docente unidocente. Todos ellos son importantes en el desarrollo de la educación; sin embargo, hay que reconocer que el sistema 
educativo posee $49,7 \%$ de escuelas unidocentes, administradas por personal docente unidocente, quienes son supervisados por sus jefes inmediatos, los Supervisores de Centros Educativos.

Como se mencionó anteriormente, el papel del personal docente unidocente debe verse la persona a cargo del desarrollo y ejecución del currículo nacional en todos los niveles del primero y segundo ciclos. Es decir, su labor pedagógica implica atender los seis grados de la Educación General Básica. Paralelamente, debe atender la parte administrativa del centro educativo, en igualdad de condiciones que el resto de instituciones educativas del país. Esto hace que la administración de la institución se ejerza en condiciones inadecuadas, dada su complejidad; en consecuencia, requieren de todo el apoyo que se les pueda brindar para desempeñar la gestión escolar en forma eficiente y eficaz, en concordancia con las políticas nacionales y las necesidades de las comunidades escolares.

La atención general de la institución y el abordaje del proceso de enseñanza y aprendizaje involucra una compleja gama de acciones que recaen en una sola persona; en este caso, en el maestro o maestra unidocentes. Desde esta perspectiva, se compromete la calidad de la educación.

El personal docente unidocente no cuenta con ningún programa de atención, asesoría, seguimiento, capacitación y control del desempeño. El único apoyo que reciben es el que brinda el Supervisor de Centros Educativos del circuito. Sin embargo, estas personas cumplen con la función de control de la gestión escolar, en asuntos meramente administrativos. En este sentido, los problemas se profundizan, ya que se exige a estos docentes el mismo volumen de trabajo que a las demás instituciones educativas del país, sin tomar en cuenta sus diferencias, limitaciones y desventajas en el ejercicio del doble cargo de docentes y administradores(as) de la educación. El personal docente unidocente, no cuenta con el apoyo necesario en la gestión curricular. Para cumplir a cabalidad con la función formadora requiere de capacitación, asesoramiento y todo tipo de formación en servicio que favorezca su desempeño pedagógico.

En esta investigación se identificó como problema la carencia de espacios formativos y de desarrollo profesional del personal docente unidocente del circuito 05, de la Regional Educativa de Santa Cruz y, considerando que la actualización es una tarea permanente, es fundamental una oferta para la actualización permanente, así como herramientas pedagógicas, 
que le sean útiles en sus labores cotidianas, con el propósito de mejorar la calidad de su práctica educativa, tanto en lo pedagógico como en la gestión administrativa.

Con base en los hallazgos del estudio, a continuación se presenta un modelo de gestión, donde se consideran los componentes relacionados con el desempeño del maestro o maestra unidocentes. El diseño de este modelo atiende dos grandes áreas de la gestión escolar: el área administrativa y el área pedagógica, tal y como se ilustra a continuación.

\section{Figura 1}

Áreas de intervención del modelo de gestión de escuelas unidocentes

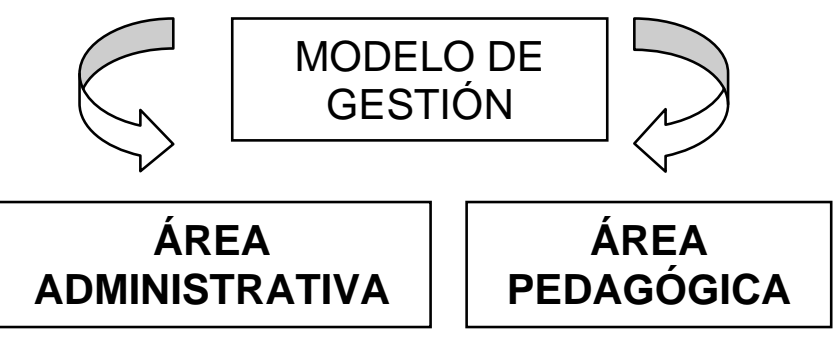

El logro o fracaso del proceso de enseñanza y aprendizaje depende de las acciones y decisiones que tome la persona que administra el centro educativo. Por tal motivo, conocer la problemática del personal docente unidocente en cuanto a su formación profesional, permite afirmar que las políticas administrativas regionales o circuitales no favorecen el mejoramiento continuo ni el desempeño de estos maestros y maestras.

Debido a que no existen espacios donde el personal docente unidocente se capacite y reciba la asesoría que requiere para atender de manera adecuada sus obligaciones, es indispensable una propuesta de intervención en estas áreas. Por ello, se propone la intervención pedagógica mediante diferentes talleres, espacios de refrescamiento y actualización, con el fin de atender las necesidades del personal docente unidocente, para ofrecerles herramientas de apoyo para la gestión administrativa y pedagógica.

Desde el enfoque constructivista, la propuesta es viable porque el personal docente unidocente necesita desarrollar su potencial en la práctica, para hacerle frente a sus carencias 
pedagógicas; y, en esa medida, se construye el conocimiento. De igual manera, requiere fortalecer la práctica administrativa. Como se argumentó anteriormente, está en manos del maestro o maestra dirigir el desempeño de la administración del centro educativo unidocente, especialmente, porque las labores administrativas son las que más tiempo y dedicación demandan.

\subsection{Acciones del Modelo de Gestión de Escuelas Unidocentes}

Tomando en cuenta los elementos implícitos, dentro de cada una de las áreas de intervención del modelo de gestión para las escuelas unidocentes, se presentan acciones que permitan este tipo de docentes realizar una labor eficiente, acorde con los objetivos y fines de la educación costarricense.

\section{a. Área Administrativa}

Esta área comprende aquellas acciones administrativas que lleva a cabo el maestro o maestra unidocente, tales como:

- Elaboración de informes.

· Elaboración del Plan Operativo Anual (POA).

- Elaboración del Proyecto Educativo Institucional (PEI).

- Elaboración del diagnóstico comunal e institucional.

- Conformación de los organismos de apoyo.

- Planificación institucional.

- Asistir a reuniones con superiores.

- Convocar reuniones con padres de familia y comités de apoyo.

- Promover la ejecución del presupuesto ordinario y el de DANEA.

- Organizar el servicio del comedor escolar.

- Evaluación de los proyectos y programas institucionales.

- Promover proyectos comunales. 
- Todas aquellas funciones inherentes al cargo de índole administrativa.

\section{b. Área Pedagógica}

Esta área comprende las acciones pedagógicas que realiza el maestro o maestra unidocente, tales como:

- Realización de diagnósticos pedagógicos a los estudiantes.

- Desarrollo de la labor docente (lecciones didácticas).

- Elaboración del planeamiento didáctico (minuta).

- Planificación pedagógica (unidades didácticas).

- Programación de reuniones con los padres de familia para asuntos pedagógicos.

- Elaboración de pruebas para la evaluación de los aprendizajes.

- Atención de las necesidades educativas de los alumnos.

- Elaboración de expedientes acumulativo de los estudiantes.

- Ejecutar acciones de otras dependencias gubernamentales (campañas de salud).

- Llenado de documentos específicos de la labor docente.

- Todas aquellas funciones inherentes al desempeño pedagógico.

\subsection{Plan de acción}

Para el desarrollo del modelo de áreas de intervención, se proponen las siguientes temáticas:

Tabla 1

Modelo de áreas de intervención

\begin{tabular}{|c|c|}
\hline Área administrativa & Área pedagógica \\
\hline $\begin{array}{l}\text { - Diseño de papelería administrativa. } \\
\text { - Formulación de la planificación } \\
\text { institucional. }\end{array}$ & $\begin{array}{ll}\text { - } & \text { Diseño de documentos de referencia } \\
& \text { estudiantil. } \\
\text { - } & \text { Organización del proceso de } \\
\text { enseñanza-aprendizaje. }\end{array}$ \\
\hline
\end{tabular}


- Organización de la labor de los órganos de apoyo.

- Organización de las sesiones con superiores.

- Organización de las sesiones institucionales.

- Diseño de la propuesta presupuestaria anual.

- Diseño de los proyectos y programas institucionales.

- Vinculación y proyección comunal.
- Organización de las sesiones de los reportes y avances pedagógicos.

- Organización de la evaluación de los aprendizajes.

- Apertura a la ejecución de labores extracurriculares.

- Formación profesional.

- Organización de espacios para formación de los padres de familia.

\section{BIBLIOGRAFÍA}

Álvarez P., Y. (2007). Repercusiones de la labor administrativa y su impacto en el desarrollo del currículo de los niños y niñas de las escuelas unidocentes Rodeíto y las delicias del circuito 04 de la Región Educativa Liberia. Seminario de investigación (licenciatura) Centro de Investigación y Docencia en Educación. Universidad Nacional. Guanacaste, Costa Rica.

Arroyo, J. A. (2001). Incidencia de los indicadores en la calidad de la educación. San José, Costa Rica: EUCR.

Barrantes, R. (2001). Investigación: un camino al conocimiento. San José, Costa Rica: EUNED.

Buendía, L., Colás, M. y Hernández, F. (2001). Métodos de investigación en psicopedagogía. Madrid: McGraw-Hill.

Bustos M., Campos J., Corea, Y., González, E. y Mendoza, L. (2006). La práctica unidocente en las escuelas de Guanacaste: una visión entre la teoría académica y su realidad en el aula. Tesis de Licenciatura en Educación Primaria. Universidad de Costa Rica. Guanacaste, Costa Rica.

Castillero, A. (2002). Gestión y supervisión en el centro de educación básica. Cartago, Costa Rica: UTEC.

Chavarría, F., Rodríguez, J., Barrantes, S. y Valle, L. (2009). Análisis de la gestión que desempeñan los educadores unidocentes del circuito 02 de Pitahaya de la Regional de Puntarenas. Seminario de Graduación para optar por el grado de Licenciatura en Ciencias de la Educación con énfasis en Administración Educativa. Universidad de Costa Rica. Puntarenas, Costa Rica. 
Chiavenato, I. (2007). Administración de los recursos humanos. Colombia: McGraw-Hill.

Contreras, C. (2006). Pertinencia de la gestión administrativa de los asesores supervisores circuitos 02 y 04, en I y II Ciclos, de la Dirección Regional de Educación de Liberia. Tesis de Maestría en Administración Educativa. Universidad de Costa Rica. Guanacaste, Costa Rica.

Consejo Nacional de Rectores [CONARE]. (2003). Noveno Informe Estado de la Nación en Desarrollo Humano Sostenible. Programa Estado de la Nación. San José, Costa Rica: CONARE.

Consejo Nacional de Rectores [CONARE]. (2009). Décimo Tercer Informe Estado de la Nación en Desarrollo Humano Sostenible. Programa Estado de la Nación. San José, Costa Rica: CONARE.

Consejo Nacional de Rectores [CONARE]. (2011). Tercer Informe del Estado de la Educación. Programa Estado de la Nación. San José, Costa Rica: CONARE.

C.R. (1957). Ley Fundamental de Educación. San José, Costa Rica: Autor.

Cortés, L. (1989). Análisis de la supervisión educativa en la dirección regional de enseñanza de Santa Cruz. Tesis de Licenciatura en Ciencias de la Educación con énfasis en Curriculum. Universidad de Costa Rica. Guanacaste, Costa Rica.

Delgado M., W. (2007). Inclusión: Principio de calidad educativa desde la perspectiva del desarrollo humano. Revista Educación, 31(2). San José, Costa Rica: Universidad de Costa Rica.

Delors, J., Mufti, I., Amagi, I., Carneiro, R., Chung, F., Geremek, B. y Nanzhao, Z. (1996). La Educación Encierra un Tesoro. Informe a la UNESCO de la Comisión Internacional sobre la Educación para el siglo XXI (Compendio). Francia: Santillana Ediciones UNESCO.

Díaz, F. (2002). Educación Unidocente desde la práctica. San José, Costa Rica: EUNA

Espinoza, R. (1987). Perfil profesional del maestro Unidocente. Departamento de Investigación Educativa. San José. Costa Rica: EUCR.

Ferreiro, R. (2006) Nuevas alternativas de aprender y enseñar; aprendizaje cooperativo. México: Trillas.

Gairín S., J. (2000). Cambio de cultura y organizaciones que aprenden. Revista Educar 27. San José, Costa Rica. 
Garbanzo, V. y Orozco, D. (2007). Desafíos del sistema educativo costarricense: un nuevo paradigma de la Administración de la Educación. Revista Educación 31 (2), 95-110. Recuperado de SIN: 0379-7082.

García L., N, Rojas P., M. y Campos S., N. (2002). La administración escolar para el cambio y el mejoramiento de las instituciones educativas. San José, Costa Rica: EUCR.

Guandara, W. (1998). Guía para orientar el proceso en las escuelas unidocentes. San José, Costa Rica: Ministerio de Educación Pública.

Guido L., K. (2006). Gestión del asesor supervisor en el mejoramiento administrativo de seis escuelas unidocentes del circuito 03 de Liberia. Tesis para optar por el grado de Maestría en Administración Educativa. Guanacaste, Costa Rica.

Guzmán, S. y Sánchez Escobedo, P. (2006). Efectos de un programa de capacitación de profesores en el desarrollo de habilidades de pensamiento crítico en estudiantes universitarios en el Sureste de México. Revista Electrónica de Investigación Educativa, 8 (2). Recuperado de http://redie.uabc.mx/vol8no2/contenido-guzman.html

H. de Materi, L. y Bähler, R. (1984). Administración escolar. Planeamiento Institucional. Buenos Aires: El Ateneo.

Hernández S., R., Fernández C., C. y Baptista L., P. (1999). Metodología de la investigación. México: McGraw- Hill.

Hernández S., R., Fernández C., C. y Baptista L., P. (2003). Metodología de la investigación. México: McGraw- Hill.

Imbernón, F.et al. (2006). Perspectivas de formación docente. Perú: Ministerio de Educación. Recuperado de ISBN 9972-854-21-3.

Lastarria Z., J.A. (2008). Formación docente, mejora de la gestión educativa y dotación de recursos en los centros educativos públicos del cono este de Lima. Supervisión y monitoria educativa. Módulos de Aprendizaje. Recuperado de http://www.ucss.edu.pe/cesed/biblio-virtual/gpedagogica/supervision_educativa_monitoreo1.pdf

Lépiz, C. (2003). La administración y la Planificación como Procesos. San José, Costa Rica: EUNED.

Likert, R. y Gibson, J. (1986). Nuevas formas para solucionar conflictos. México: Trillas. 
Martínez L., E. (setiembre, 2006). La dirección como un factor clave de eficacia y de cambio escolar. Revista Avances en supervisión educativa (4). España: Asociación de Inspectores de Educación de España.

Masís, C. (1990). Administración de instituciones o procesos educativos. San José, C.R.: EUNED.

Ministerio de Educación Pública [MEP]. (1957). Ley Fundamental de Educación. San José, Costa Rica: MEP.

Ministerio de Educación Pública [MEP]. (2005). Manual Descriptivo de Clases de Puestos Docentes del Régimen de Servicio Civil. San José, Costa Rica: MEP.

Ministerio de Educación Pública [MEP]. (2008). El centro educativo de calidad como eje de la educación. San José, Costa Rica: MEP.

Ministerio de Educación Pública [MEP]. (2009). Compendio de Normas Reguladoras para el Desarrollo Curricular. San José, Costa Rica: MEP.

Moreno B., M. G. (1995). Investigación e Innovación Educativa. Revista Electrónica La Tarea, 7, Recuperado de http://www.latarea.com.mx/articu/articu7/bayardo7.htm

Mosley, D., Megginson, L., y Pietri, P.H. (2005). Supervisión. La práctica del empowerment, desarrollo de equipos de trabajo y motivación. México: Thomson Editores.

Muñoz, J. F., Quintero, J. y Munévar, R. A. (2002). Experiencias en investigación-acciónreflexión con educadores en proceso de formación en Colombia. Revista

Electrónica de Investigación Educativa, 4(1). Recuperado de http://redie.uabc.mx/contenido/vol4no1/contenido-munevar.pdf

Nérici, I. (1975). Introducción a la Supervisión Escolar. Buenos Aires: Kapeluz.

Ogando, F. (2005). Reporte sobre Estado de la Investigación en Gestión Escolar. Práctica Pedagógica y Calidad. Programa Estado de la Nación. San José, Costa Rica: CONARE.

Palladino, E. (2005). Diseños curriculares y calidad educativa. Buenos Aires: Espacio Editorial. 
Pérez B., M. I. (1998). Incidencia de los asesoramientos impartidos por los asesores específicos de la Unidad de Desarrollo Educativo de Dirección Regional de San Ramón, en el desempeño de los docentes de materias básicas e idiomas de secundaria del Circuito 07. Tesis para optar por el grado de Maestría en Administración Educativa. Universidad de Costa Rica. San José, Costa Rica.

Pineda H., P. (2000). Evaluación del impacto de la formación en las organizaciones. Revista Educar, 27, 119-133. Recuperado de http://ddd.uab.cat/pub/educar/0211819Xn27p119.pdf

Rivas B., J.J. (2006). Calidad y eficiencia de la gestión del asesor supervisor en el proceso de la planificación curricular de las escuelas unidocentes período 2005-2006. Tesis para optar por el grado de Maestría en Administración Educativa. Universidad de Costa Rica. Liberia, [Guanacaste], Costa Rica.

Rodríguez G., R. (2007). Reflexiones sobre cultura organizacional e instituciones en el mundo actual. Revista Electrónica Psicología Científica.com. Recuperado de http://www.psicologiacientifica.com/bv/psicologia-271-1-reflexiones-sobre-culturaorganizacional-e-instituciones-en-.html

Segura, M. (2008). Pasos hacia una evaluación curricular en las instituciones educativas. San José, Costa Rica: EUCR.

Segura S., C. (2002). La Calidad de la Educación vista en los programas de estudio de las asignaturas y el Liderazgo del Director: Un caso para reflexionar en la Toma de Decisiones. Seminario de Proyecto de Graduación para optar por el grado de Maestría en Administración de la Educación. Universidad Estatal a Distancia. San José, Costa Rica.

Torres V., N. y Céspedes M., E. (2005). Acerca de la Educación RURAL en Costa Rica. Heredia, Costa Rica: UNA.

Ugalde, J. (1979). Administración institucional 1. San José, Costa Rica: EUNED.

Vargas J, I. (2008). Análisis de cinco desafíos en el ejercicio de la administración educativa. Actualidades Investigativas en Educación, 8(1). San José, Costa Rica: INIEUniversidad de Costa Rica.

Venegas J., P. (1999). Algunos elementos de investigación. San José, Costa Rica: EUNED.

Venegas J., P. (2000). Simposio Interinstitucional sobre Legislación Educativa. San José, Costa Rica: Universidad de Costa Rica.

Zepeda, F. (1999). Psicología organizacional. México: Addison Wesley Longman. 